\title{
A FORMULA ON THE SUBDIFFERENTIAL OF THE DECONVOLUTION OF CONVEX FUNCTIONS
}

\author{
M. VOLLE
}

It is known that, under suitable assumptions, the subdifferential $\partial(f \square g)$ of the infimal convolution of two convex functions $f$ and $g$ coincides with the parallel sum of $\partial f$ and $\partial g$. We prove that a similar formula holds for the subdifferential of the deconvolution of two convex functions: under appropriate hypothesis it coincides with the parallel star-difference of the sub-differentials of the functions.

\section{Preliminaries}

In what follows $(X, Y)$ is a couple of locally convex real topological spaces paired in separating duality by a bilinear form $\langle.,$.$\rangle , and \Gamma_{0}(X)\left(\right.$ respectively $\Gamma_{0}(Y)$ ) is the class of convex, lower-semicontinuous proper functions defined on $X$ (respectively $Y$ ) with values in $\mathbb{R} \cup\{+\infty\}$. Given $h, k: X \rightarrow \overline{\mathbb{R}}$, the inf-convolution $h \square k$ is defined by

$$
(h \square k)(x)=\inf _{u \in X}\{h(x-u) \dot{+} k(u)\} \quad \text { for all } x \in X,
$$

where $\dot{t}$ is the upper extension of the addition to $\overline{\mathbb{R}}$ (that is, $(+\infty) \dot{+}(-\infty)=$ $(+\infty) \dot{-}(+\infty)=+\infty$, see $[10])$.

The deconvolution, denoted by the symbol $\theta$, is a kind of inverse operation for the inf-convolution. It was introduced by Hiriart-Urruty and Mazure [5] in order to solve the inf-convolution equation

$$
\text { find } \quad \xi \in \overline{\mathbb{R}}^{X} \quad \text { such that } \quad k \square \xi=h .
$$

It is known [9, Corollary 2] that a solution to (1) exists if and only if the function

$$
x \longmapsto(h \boxminus k)(x)=\sup _{u \in X}\{h(x+u)-k(u)\}
$$

is one of them. The function defined by (2) is referred to as the deconvolution of $h$ and $k$. Here the symbol - denotes the lower extension of the subtraction to $\overline{\mathbb{R}}$ (that is, $(+\infty)-(+\infty)=(+\infty)+(-\infty)=-\infty,[10])$

Received 27th April, 1992

Copyright Clearance Centre, Inc. Serial-fee code: $0004-9729 / 93 \$ A 2.00+0.00$. 
The operation $\boxminus$ has many interesting applications. For instance, taking the deconvolution of convex quadratic forms yields a variational formulation of the parallel subtraction of matrices and operators (for example $[8,13]$ ).

The deconvolution operation is strongly linked to the star-difference of sets. Recall that the star-difference of two subsets $A$ and $B$ of a linear space $E$ is defined by

$$
\begin{gathered}
A^{*}-B=\{x \in E: x+B \subset A\} . \\
E(f)=\{(x, r) \in X \times \mathbb{R}: f(x) \leqslant r\}
\end{gathered}
$$

for the epigraph of $f \in \overline{\mathbb{R}}^{E}$ and $I_{C}$ for the indicator function of $C \subset X \quad\left(I_{C}(x)=0\right.$ if $x \in C, I_{C}(x)=+\infty$ if $\left.x \in E \backslash C\right)$, we have then [15, Proposition 6]

$$
\begin{gathered}
E(h \boxminus k)=E(h)^{*}-E(k) \\
I_{A} \boxminus I_{B}=I_{A-B}^{*} \quad \text { if } B \neq \emptyset .
\end{gathered}
$$

In the context of epigraphical analysis [1], formula (3) suggests another terminology for the deconvolution operation, namely the epigraphical difference or, better, the epigraphical star-difference.

In connection with Fenchel's duality theory, the deconvolution operation enjoys some noteworthy properties. Recall that the Fenchel conjugate of $f \in \overline{\mathbb{R}}^{X}$ is defined by

$$
f^{*}(y)=\sup _{x \in X}\{\langle x, y\rangle-f(x)\} \quad \text { for all } y \in Y .
$$

In a similar way one defines the conjugate of a function in $\overline{\mathbb{R}}^{Y}$. A fundamental result concerning the conjugacy operation is that

$$
f=f^{* *} \text { for all } f \in \Gamma_{0}(X) .
$$

Now, according to Hiriart-Urruty [4, Theorem 2.2], the formula

$$
\left(h^{*} \dot{-} k^{*}\right)^{*}=h \boxminus k
$$

holds for all $h, k \in \Gamma_{0}(X)$. It follows that

$$
(h \boxminus k)^{*}=\left(h^{*} \dot{-} k^{*}\right)^{* *} \text {. }
$$

If $h^{*} \dot{-} k^{*}$ turns out to be in $\Gamma_{0}(Y)$, one can also write

$$
(h \boxminus k)^{*}=h^{*}-k^{*} .
$$


We end this section by recalling some facts and by introducing some notation. For any extended-real-valued function $\xi \in \mathbb{\mathbb { R }}^{Y}$, the $\varepsilon$-subdifferential $(\varepsilon \geqslant 0)$ of $\xi$ at $y \in \xi^{-1}(\mathbb{R})$ is the set

$$
\partial_{e} \xi(y)=\{x \in X: \forall v \in Y: \xi(v)-\xi(y) \geqslant\langle x, v-y\rangle-\varepsilon\} .
$$

For $\varepsilon=0$ we set, as usual, $\partial_{0} \xi(y)=\partial \xi(y)$. In connection with this concept, the following classical property will be used later on (see for example [6, p.351]):

LEMma 1. For any $\xi \in \overline{\mathbb{R}}^{Y}$ and $y \in Y$ such that $\xi(y)=\xi^{* *}(y) \in \mathbb{R}$ one has

$$
\partial \xi(y)=\partial \xi^{* *}(y) \text {. }
$$

Proof: As we always have $\xi^{* *} \leqslant \xi$, the inclusion $\supset$ is obvious. Now, for any $x \in \partial \xi(y)$, the affine continuous form $\langle x,\rangle-.\langle x, y\rangle+\xi^{* *}(y)$ is smaller than $\xi$. As $\xi^{* *}$ coincides with the upper hull of all affine continuous minorants of $\xi$, we have $\langle x,\rangle-.\langle x, y\rangle+\xi^{* *}(y) \leqslant \xi^{* *}$, that is to say, $x \in \partial \xi^{* *}(y)$.

The directional derivative of a function $\xi \in \overline{\mathbb{R}}^{Y}$ at a point $y \in \xi^{-1}(\mathbb{R})$ is defined, when it exists, by

$$
\xi^{\prime}(y, d)=\lim _{t \rightarrow 0_{+}} t^{-1}(\xi(y+t d)-\xi(y)) \quad \text { for all } d \in Y ;
$$

the lower subdifferential of $\xi$ at $y$ is the set (for example [14])

$$
\partial^{-} \xi(y)=\left\{x \in X:\langle x, .) \leqslant \xi^{\prime}(y, .)\right\} .
$$

The above set obviously contains $\partial \xi(y)$ and coincides with $\partial \xi(y)$ when $\xi$ is convex; in that case ( $\xi$ convex) it is well known (for example $[6, p .354]$ ) that $\xi^{\prime}(y,$.$) is a sublinear$ function whose Fenchel conjugate is the indicator function of $\partial \xi(y)$; in other words

$$
\left(\xi^{\prime}(y, .)\right)^{*}=I_{\theta \xi(y)} .
$$

If, moreover, $\xi$ is continuous at $y$, then $\xi^{\prime}(y,$.$) is finitely valued, continuous, and one$ has

$$
\xi^{\prime}(y, .)=\left(I_{\theta \xi(y)}\right)^{*}
$$

2. ON THE SUBDIFFERENTIAL OF THE DIFFERENCE OF TWO CONVEX FUNCTIONS

Let $\varphi$ and $\psi$ in $\overline{\mathbb{R}}^{Y}$ be two convex functions, finite at the point $y \in Y$. In connection with the subdifferentiability of the difference $\varphi-\psi$, there are two formulas worth mentioning:

$$
\begin{gathered}
\partial(\varphi \dot{-} \psi)(y)=\bigcap_{\epsilon>0} \partial_{\epsilon} \varphi(y)^{*}-\partial_{\epsilon} \psi(y) \\
\partial^{-}(\varphi-\psi)(y)=\partial \varphi(y)^{*}-\partial \psi(y) .
\end{gathered}
$$


The first one is due to Martinez-Legaz and Seeger [7, Theorem 1] and applies to arbitrary functions $\varphi$ and $\psi$ in $\Gamma_{0}(Y)$; the second one has been mentioned by Ellaia [3, p.94] for $\varphi$ and $\psi$ convex on $\mathbb{R}^{n}$ and finitely valued. The next lemma extends the second formula to our general setting.

Lemma 2. Let $\varphi, \psi \in \overline{\mathbb{R}}^{Y}$ be convex functions, finite at $y \in Y$, and assume that $\psi$ is continuous at $y$. Then

$$
\partial^{-}(\varphi-\psi)(y)=\partial^{-}(\varphi-\psi)(y)=\partial \varphi(y) \stackrel{*}{-} \partial \psi(y)
$$

Proof: Let us consider only the case - . Each of the following lines is equivalent to $x \in \partial \varphi(y) \stackrel{*}{-} \partial \psi(y)$ :

$$
\begin{aligned}
& \forall u \in \partial \psi(y): x+u \in \partial \varphi(y) \\
& \forall u \in \partial \psi(y):\langle x+u, .\rangle \leqslant \varphi^{\prime}(y, .) \quad\left(\text { as } \partial \varphi(y)=\partial^{-} \varphi(y)\right) \\
& \langle x, .\rangle+\left(I_{\partial \psi(y)}\right)^{*} \leqslant \varphi^{\prime}(y, .) \quad(\text { by taking the supremum for } u \in \partial \psi(y)) \\
& \langle x, .\rangle+\psi^{\prime}(y, .) \leqslant \varphi^{\prime}(y, .) \quad(\text { from }(9)) \\
& \langle x, .) \leqslant \varphi^{\prime}(y, .)-\psi^{\prime}(y, .) \quad\left(\text { as } \psi^{\prime}(y, .) \text { is finitely valued }\right) \\
& \langle x, .\rangle \leqslant(\varphi-\psi)^{\prime}(y, .) \\
& x \in \partial^{-}(\varphi-\psi)(y) .
\end{aligned}
$$

Before passing to next section we record here a by-product of this lemma.

Corollary . Let $\varphi, \psi$ be in $\Gamma_{0}(Y)$; assume that $\varphi \dot{-} \psi$ is convex, finite at $y$, and $\psi$ is continuous at $y$; then

$$
\bigcap_{\varepsilon>0} \partial_{\varepsilon} \varphi(y) \stackrel{*}{-} \partial_{\varepsilon} \psi(y)=\partial \varphi(y) \stackrel{*}{-} \partial \psi(y)
$$

Proof: As $\partial^{-}(\varphi \dot{-} \psi)=\partial(\varphi \dot{-} \psi)$, it suffices to apply formula (10) and Lemma 2.

3. ON THE SUBdifferential OF THE DECONVOLUTION

Let us present now the main results of this note. Given $h, k \in \Gamma_{0}(X)$, the parallel sum of $\partial h$ and $\partial k$ is defined by (see for example [12])

$$
\partial h \square \partial k=\left((\partial h)^{-1}+(\partial k)^{-1}\right)^{-1} .
$$


Here $(\partial h)^{-1}$ is set for the inverse of the multivalued operator $\partial h$; in other words:

$$
y \in(\partial h \square \partial k)(x) \Leftrightarrow x \in(\partial h)^{-1}(y)+(\partial k)^{-1}(y)
$$

where + denotes the Minkowski vectorial addition. It turns out that, under appropriate constraint qualifications $[11,12]$, the formula $\partial(h \square k)=\partial h \square \partial k$ holds. It is tempting to ask wether or not there is a similar formula for the deconvolution operator. To this end, let us introduce the notion of parallel star difference for subdifferentials.

Definition: Let $h$ and $k$ be in $\Gamma_{0}(X)$. The parallel star difference of the subdifferentials $\partial h$ and $\partial k$ is the multivalued operator $\partial h \boxminus \partial k$ defined by

$$
\partial h \boxminus \partial k=\left((\partial h)^{-1}-(\partial k)^{-1}\right)^{-1},
$$

that is, for any $(x, y) \in X \times Y$,

$$
y \in(\partial h \boxminus \partial k)(x) \Leftrightarrow x \in(\partial h)^{-1}(y) \stackrel{*}{-}(\partial k)^{-1}(y) .
$$

In [5, Proposition 7] one finds a lower estimate for the subdifferential of two finitely valued convex functions $f, g$ on $\mathbb{R}^{n}$; namely it is shown that

$$
\partial(g \boxminus f)(x) \supset \bigsqcup_{\left(x_{1}, x_{2}\right) \in A x} \partial g\left(x_{1}\right) \cap \partial f\left(x_{2}\right),
$$

where $A x=\left\{\left(x_{1}, x_{2}\right) \in \mathbb{R}^{n} \times \mathbb{R}^{n}: x=x_{1}-x_{2},(g \boxminus f)(x)=g\left(x_{1}\right)-f\left(x_{2}\right)\right\}$. As pointed out to me by A. Seeger, the condition $\left(x_{1}, x_{2}\right) \in A x$ yields the inclusion $\partial g\left(x_{1}\right) \subset$ $\partial f\left(x_{2}\right)$. Moreover, the convexity assumption on $f$ and $g$ is superfluous:

Proposition. Let $f, g$ be arbitrary extended real valued functions on $X$. Then, for any $x \in X$, we have

$$
\partial(g \boxminus f)(x) \supset \bigsqcup_{v \in E(x)} \partial g(v),
$$

where $E(x)=\{v \in X: g(v)-f(v-x)=(g \boxminus f)(x) \in \mathbb{R}\}$.

Proof: Let $v$ be in $E(x)$ and $y \in \partial g(v)$; then $g(v)$ and $f(v-x)$ are real numbers and we have,

$$
(g \boxminus f)(z) \geqslant g(z+v-x)-f(v-x) \quad \text { for all } z \in X .
$$

Hence,

$$
(g \boxminus f)(z)-(g \boxminus f)(x) \geqslant g(z+v-x)-f(v-x)-g(v)+f(v-x) \quad \text { for all } z \in X,
$$

and finally

$$
(g \boxminus f)(z)-(g \boxminus f)(x) \geqslant g(z+v-x)-g(v) \geqslant\langle z-x, y\rangle \quad \text { for all } z \in X .
$$


This shows that $y \in \partial(g \boxminus f)(x)$.

The next result provides an upper estimate for the subdifferential of the deconvolution of two convex functions $h, k \in \Gamma_{0}(X)$ in terms of the parallel star difference of $\partial h$ and $\partial k ;$ it involves the set

$C(h, k)=\left\{y \in Y: k^{*}\right.$ is finite and continuous at $y$, and $\left.\left(h^{*}-k^{*}\right)(y)=\left(h^{*}-k^{*}\right)^{* *}(y)\right\}$

THEOREM 1. Let $X, Y$ be locally convex spaces in separating duality, and let $h, k \in \Gamma_{0}(X)$. Then, for all $x \in X$, we have

$$
\partial(h \boxminus k)(x) \cap C(h, k) \subset(\partial h \boxminus \partial k)(x) .
$$

Proof: Assume that $y \in \partial(h \boxminus k)(x) \cap C(h, k)$. We have to show that $x \in$ $(\partial h)^{-1}(y)^{*}-(\partial k)^{-1}(y)$. As $y \in \partial(h \boxminus k)(x)$ we have $x \in \partial(h \boxminus k)^{*}(y)$. Now, from (6), $(h \boxminus k)^{*}=\left(h^{*}-k^{*}\right)^{* *}$; then $x \in \partial\left(h^{*}-k^{*}\right)^{* *}(y)$; as $y \in C(h, k)$ it follows from Lemma 1 that $x \in \partial\left(h^{*}-k^{*}\right)(y)$ and, a fortiori, $x \in \partial^{-}\left(h^{*}-k^{*}\right)(y)$. So, by Lemma 2, we obtain $x \in \partial h^{*}(y) \stackrel{*}{-} \partial k^{*}(y)$.

Let us give an example showing that Theorem 1 cannot be improved without additional assumptions. Take for $X$ an Hilbert space with closed unit ball $B, h=$ \|\|$, k=\left(\|\|^{2}\right) / 2$. We have then by $(6)(h \boxminus k)^{*}=\left(I_{B}-\left(\|\|^{2}\right) / 2\right)^{* *}=I_{B}-$ $1 / 2$ so that $h \boxminus k=\|\|+1 / 2$. Note also that $C(h, k)=\{y \in X:\|y\| \geqslant 1\}$. For the subdifferentials we have, on one hand,

$$
\partial(h \boxminus k)(x)=\left\{\begin{array}{lll}
\frac{x}{\|x\|} & \text { if } & x \neq 0 \\
B & \text { if } & x=0
\end{array}\right.
$$

and, on the other hand, $y \in(\partial h \boxminus \partial k)(x)$ if and only if

$$
x \in \partial I_{B}(y)-\partial\left(\frac{\|\|^{2}}{2}\right)(y)=\partial I_{B}(y)-y=\left\{\begin{array}{lll}
\emptyset & \text { if } & \|y\|>1 \\
l-1,+\infty l y & \text { if } & \|y\|=1 \\
-y & \text { if } & \|y\|<1
\end{array} .\right.
$$

In particular,

$$
\begin{aligned}
\text { for } \quad\|x\|=1 & \partial(h \boxminus k)(x)=\{x\} \underset{f}{f}(\partial h \boxminus \partial k)(x)=\{x,-x\} \\
\text { for } \quad x=0 & \partial(h \boxminus k)(0)=B \supsetneqq(\partial h \boxminus \partial k)(0)=\{y:\|y\|=1\} \cup\{0\} .
\end{aligned}
$$

With stronger assumptions it is possible to give an exact formula for $\partial(h \boxminus k)$ : 
THEOREM 2. Let $X, Y$ be locally convex spaces paired in separating duality, and let $h, k \in \Gamma_{0}(X)$. Assume that $k^{*}$ is finite and continuous over $Y$ and that $h^{*}-k^{*}$ is convex. Then,

$$
\partial(h \boxminus k)=\partial h \boxminus \partial k .
$$

Proof: Since for each $f \in \Gamma_{0}(X)$ one has $(\partial f)^{-1}=\partial f^{*}$, we easily obtain the equivalence between the assertions below:

$$
\begin{aligned}
& y \in \partial(h \boxminus k)(x) \\
& x \in \partial(h \boxminus k)^{*}(y) \\
& x \in \partial\left(h^{*}-k^{*}\right)(y) \quad\left(\text { by }(5) \text { as } h^{*}-k^{*}\right. \text { is convex proper lower semicontinuous) } \\
& x \in \partial h^{*}(y)^{*} \partial k^{*}(y) \quad(\text { from Lemma 2) } \\
& x \in(\partial h)^{-1}(y) \stackrel{-}{-}(\partial k)^{-1}(y) .
\end{aligned}
$$

EXAmple: Let us take for $X$ a Hilbert space, $h \in \Gamma_{0}(X), k \in \Gamma_{0}(X)$. Assume that $k^{*}$ is finite over $X$ (hence continuous) and suppose that $h^{*}-k^{*}$ is strongly convex: there exists $t>0$ and $f \in \Gamma_{0}(X)$ such that $h^{*}-k^{*}=f^{*}+\left(t\|\|^{2}\right) / 2$. We have then by (5)

$$
h \boxminus k=\left(f^{*}+\frac{t\|\|^{2}}{2}\right)^{*}=f \square \frac{\|\|^{2}}{2 t} .
$$

So, $h \boxminus k$ coincides with the Moreau-Yosida regularisation of $f \in \Gamma_{0}(X)$ (for example [2, p.195]). It follows that $h \square k$ is continuously differentiable. As $h^{*}$ is also strongly convex, $h$ is continuously differentiable and we have, applying Theorem 2 ,

$$
\nabla(h \boxminus k)=\nabla h \boxminus \partial k .
$$

\section{REFERENCES}

[1] H. Attouch and R.J.B. Wets, 'Epigraphical analysis', in Analyse non linaire, (H. Attouch, J.-P. Aubin, F.H. Clarke, I. Ekeland, Editors) (Gauthier-Villars, Paris, 1989), pp. 73-100.

[2] J.-P. Aubin and I. Ekeland, Applied nonlinear analysis (Wiley, New York, 1984).

[3] R. Ellaia, Contribution à l'analyse et l'optimisation de difference de fonctions convexes, Thèse (Université Paul Sabatier, Toulouse, 1984).

[4] J.-B. Hiriart-Urruty, 'A general formula on the conjugate of the difference of functions', Canad. Math. Bull. 29 (1986), 482-485. 
[5] J.-B. Hiriart-Urruty and M.-L. Mazure, 'Formulations variationnelles de l'addition parallèle et de la soustraction parallèle d'opérateurs semi-définis positifs', C.R. Acad. Sci. Paris Série I 302 (1986), 527-530.

[6] P.-J. Laurent, Approximation et optimisation (Hermann, Paris, 1972).

[7] J.E. Martinez-Legaz and A. Seeger A formula on the approximate subdifferential of the difference of convex functions, Bull. Austral. Math. Soc. 45 (1992), 37-41.

[8] M.-L. Mazure, 'La soustraction parallèle d'opérateurs interpretée comme déconvolution de formes quadratiques convexes', Optimization 18 (1987), 465-484.

[9] M.-L. Mazure and M. Volle, 'Equations inf-convolutives et conjugaison de Moreau-Fenchel', Ann. Fac. Sci. Toulouse Math 12 (1991), 103-126.

[10] J.-J. Moreau, 'Inf-convolution, sous-additivité, convexité des fonctions numériques', $J$. Math. Pures Appl. 49 (1970), 109-154.

[11] A. Moudaf, Convolution des opérateurs monotones et convergence variationnelle (Université de Clermont-Ferrand II, 1991). (Preprint).

[12] G.B. Passty, 'The parallel sum of nonlinear monotone operators', Nonlinear Anal. 10 (1986), 215-227.

[13] E.L. Pekarev and L. Smul'jan, 'Parallel addition and parallel subtraction of operators', Math. USSR-Izv. 10 (1976), 351-370.

[14] J.-P. Penot, 'Calcul sous-différentiel et optimisation', J. Funct. Anal. 27 (1978), 248-276.

[15] M. Volle, 'Concave duality : application to problems dealing with difference of functions', Math. Programming 41 (1988), 261-278.

University of Avignon

33 rue L. Pasteur

84000 Avignon

France 